\title{
Motorcycle Lateral Dynamic Estimation and Lateral Tire-Road Forces Reconstruction Using Sliding Mode Observer
}

\author{
H. Slimi, H. Arioui and S. Mammar
}

\begin{abstract}
Extensive researches have shown that most of road accidents occur as a result of driver errors. A close examination of accident data reveals that losing the vehicle control is responsible for a huge proportion of car accidents. This observation is even more valid on motorcycle, recall that, last decade about 1600 french people are killed each year [7]. Preventing such kind of accidents using vehicle control systems, requires certain input data concerning vehicle dynamic parameters and vehicle road interaction. Unfortunately, some parameters like tire-road forces and lateral speed, which have a major impact on vehicle dynamics, are difficult to measure. Therefore, this data must be estimated. Due to the system nonlinearities and unmodeled dynamics, an observer based on sliding technique is proposed in this paper.

The estimation process method use the motorcycle dynamic response's and standard sensors cheap and easily-available. Observer performances are tested and compared to the motorcycle model responses in different simulation conditions, various initial conditions and measurement noise. The effectiveness of the present approach is make out through the simulation results and show its practical potential as a low-cost solution for calculating lateral-tire forces and motorcycle lateral speed in a accuarate way.
\end{abstract}

Index Terms-Sliding mode observer, motorcycle dynamics, tire-road contact, virtual sensors.

\section{INTRODUCTION}

$\mathbf{S}$ EVERAL studies show that major road accidents are due to human error while driving. Most drivers have little knowledge about the dynamic behavior, for this, driving assistance systems are essential key to better control their vehicle. In this context, several active safety systems ADAS ${ }^{1}$ exist, such as $\mathrm{ABS}^{2}, \mathrm{ESP}^{3}$ control type trajectory or ESC

4 for motorcycles.

A better understanding of the vehicle dynamic variables, improves the effectiveness of driving assistance systems. Longitudinal velocity, acceleration, roll angle, yaw velocity, steering angle for a two-wheeled vehicle or the suspension deflections are easily measured using low cost sensors actually installed on a wide range of newer vehicles. Contrariwise, other parameters are relevant for improving driver safety and maneuverability, namely tire-road contact forces and lateral velocity (or sideslip angle) are measured with very expensive sensors, which are unlikely to occur in future on

H. Slimi, H. Arioui and S. Mammar are with IBISC-CNRS EA 4256 Laboratory of Evry Val d'Essonne University, 91020 Evry Cedex, France hamid.slimi@ibisc.univ-evry.fr

${ }^{1}$ Advanced Driver Assistance Systems

${ }^{2}$ antilock braking systems

${ }^{3}$ Electronic Stability Program

${ }^{4}$ Electronic Stability Control ordinary vehicles for both economic and technical reasons. This justifies the interest of using the virtual sensor approach also called software sensors.

The vehicle motion is governed generally by forces generated by tires-road contact. Knowing these efforts helps to predict the motorcycle trajectory and / or unbalancing case. Thus, in curve situation, sideslip angles are established then the tires are product (subjected to) lateral forces fulfill the desired trajectory.

A characteristic curve of the transversal behavior of a tire is shown in Figure 1. We can distinguish three regions: linear with a proportionality coefficient $\mathrm{C}$, transient and saturation or sliding region. In normal driving conditions, when the tire is lightly loaded (linear regime: small sideslip and camber angles), the vehicle responds in a predictable manner to driver commands. Contrariwise, when the vehicle is subjected to high lateral forces, efforts are saturated hence the vehicle reached its stability/equilibrium limit (the control loss can be produced at any time). Thus, accurate data on these efforts and the side slip angle, leading to a better evaluation of the road friction, the possible trajectories of the vehicle and enable the development of a diagnostic tool capable to predict risks of accidents involving dangerous maneuvers.

The state of the art on observation techniques is rich.

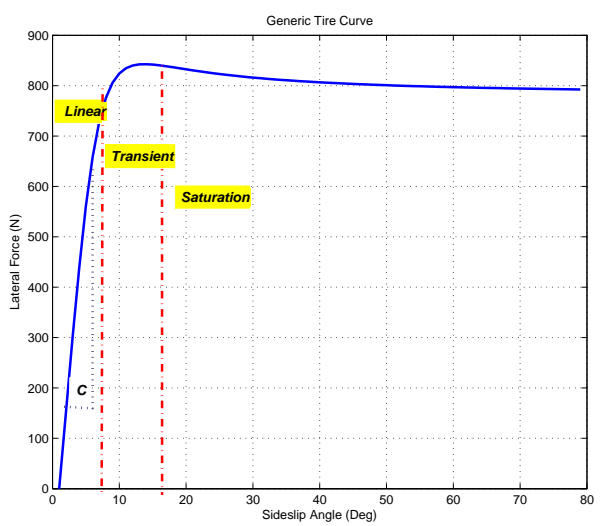

Fig. 1. Lateral force characteristics

In fact, many studies were devoted to the estimation of dynamic variables of a car, but, at the authors knowldege, no studies in this context were conducted for the motorized two-wheeled vehicles. The study presented in this paper is based primarily on a state of the art observers for estimating the dynamic automotive and reconstruction efforts at the tire- 
road contact. Indeed, Several studies have been conducted to estimate this last component, the side slip angle/rate and the road friction. For example, in [2], the authors estimate the dynamic parameters, using a complex model of 9 degrees of freedom (DoF). Further reaserchers consider first the longitudinal and lateral speed, then they deduce the lateral forces and sideslip angle. In [4] and [5], the sideslip estimator has been widely discussed. More recently, in [6], the authors estimate the lateral force by train and not at each wheel. Other studies, develop observers tire-road efforts, but they use an information on the wheels torque requiring high-cost sensors and difficult implementation.

This paper is dedicated to the estimation of the motorcycle lateral forces at each wheel and the lateral velocity of the center of gravity point. Moreover, the estimation process has been developed only on the basis of available information giving by standard sensors. The second section of this work presents the estimation process. Section 3 presents the vehicle model and tire-road dynamics. After, we describe the observer and present the observability study in section 4. The fifth section provides various simulation results and a discussion of estimation results. Finally, we give conclusions and perspectives.

\section{Estimation Process Description}

The estimation process is shown in its entirety by the block diagram in figure 2, where $a_{x}$ and $a_{y}$ are respectively the longitudinal and lateral accelerations, $\psi$ is the yaw rate, $\omega_{f}$ and $\omega_{r}$ is the wheels velocity. $z$ and $y$ are indices used to define respectively the forces axis projection (normal and lateral). $f$ and $r$ are indices used respectively for front and rear wheels. $F_{i}$ is the force representing the tire-road contact resolution and $V_{y}$ is the lateral speed at the center of gravity (cog).

In the general case, the Pacejka model calculates forces due tire-road contact (in steady state without taking into account the relaxation effect) based on dynamic states of the motorcycle model. This interconnection, between the two models, cause difficulties into the observability of the whole system due to the nonlinearity and especially the complex formulation of the Pacejka model. On the other hand, the observation of input affine systems has been widely discussed in literature. These systems category are described by the following state space system:

$$
\left\{\begin{array}{l}
\dot{x}(t)=f(x(t))+g(x(t)) \cdot u(t) \\
y(t)=h(x(t), u(t))
\end{array}\right.
$$

While our interconnected system is governed by the following state space system :

our system is initially represented by the system Equation (2), however, for ease of synthesis observer, this system will be transformed into a linear parameter varying form (LPV), details will be given in the following sections. The estimation process consists of two blocks and its role is to estimate normal and lateral forces at each tire/road level and then to evaluate the lateral friction coefficient $\left(\mu_{y f}\right.$ and $\left.\mu_{y r}\right)$. The following measurements are needed:

- yaw rate $\dot{\psi}$, roll rate $\dot{\phi}$, longitudinal $a_{x}$ and lateral $a_{y}$ accelerations measured by an inertial sensor ;

- steering angle $\delta$ measured by an optical sensor ;

- rotational velocity $\left(\omega_{f}\right.$ and $\left.\omega_{r}\right)$ for each tire given by ABS.

The first block aims to provide the vehicle's lateral forces calculated by the Pacejka model and normal tire forces using the speed and sideslip angles in tire-road contact. In this paper, we focuse only on the second block, whose main role is to estimate lateral tire forces and lateral speed. The second block makes use of the data (inputs) provided by the first block for exemple the lateral forces given by Pacejka model. One particularity of this estimation process is the use of blocks in series. By using cascaded observers, the observability problems led by an inappropriate use of the complete modeling equations are avoided enabling the estimation process to be carried out in a simple and practical way.

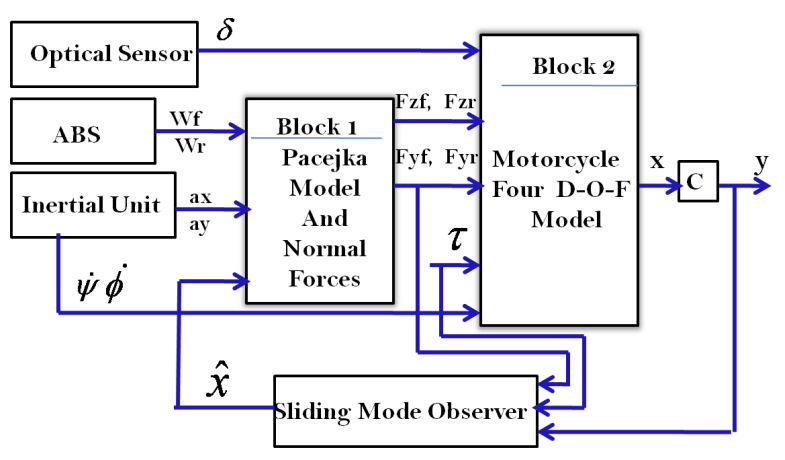

Fig. 2. Process Estimation Diagram

\section{MotorCyCle-RoAd Model}

A Motorcycle is a complex system, analytical models that describe it, can have a very important number of variables. In this case, it is necessary to reduce the complexity to light calculations and consider a real-time implementation. In this study, we use the lateral Motorcycle model and we adopt the following simplified assumptions:

- longotudinal dynamics is omitted,

- longitudinal speed is considered as constant parameter.

1) Vehicle Description and Lateral Model Equations: Important terms describing motorcycle dynamics are : lateral, yawing, rolling, and steering motions. By considering these four-degrees-of-freedoms, we can describe the fundamental characteristics of motorcycle dynamics. This study uses nonlinear four degree-of-freedom model. Figure 3 illustrates the schematic diagram for the mathematical model. The direction of the $Z$-axis in this model is chosen to be the same as that of gravitational force, $X$-axes and $Y$-Axis of the ground coordinate system lie in the road. The motorcycle model consists of two major parts: the main frame, including the rider body, and the front frame reprsenting the steering 
system, [3], [8], [9], Equations (2) through (5) describe equations of motion:

- Lateral equilibrium equation:

$$
\begin{aligned}
& M \ddot{y}+a_{2} \ddot{\phi} \cos (\phi)+M \dot{x} \dot{\psi}= \\
& \left(Y_{f}+Y_{r}\right)-C_{y} \dot{y}^{2}-M g \cos \left(\phi_{p}\right) \sin \left(\phi_{r}\right)
\end{aligned}
$$

- Roll motion equation:

$$
\begin{aligned}
& I_{x x} \ddot{\phi}+I_{x z} \ddot{\psi} \cos (\phi)+a_{2} \ddot{y}+c_{4} \dot{x} \dot{\psi}= \\
& \left(b_{1} g-\eta F_{z f}\right) \sin \delta+a_{2} g \sin (\phi)
\end{aligned}
$$

- Yaw motion equation:

$$
\begin{aligned}
& I_{z z} \cos (\phi) \ddot{\psi}+I_{x z} \ddot{\phi}= \\
& \left(L_{f} Y_{f}-L_{r} Y_{r}\right) \cos (\phi)-\tau \cos (\varepsilon)
\end{aligned}
$$

- Steering fork motion equation:

$$
\begin{aligned}
& I_{S} \ddot{\delta}-c_{5} \dot{x} \dot{\phi}+p_{2} \dot{x} \dot{\psi} \cos (\phi)+\kappa \dot{\delta}= \\
& \left(b_{1} g-\eta F_{z f}\right) \sin (\phi+\sin (\varepsilon) \sin (\delta))-\eta Y_{f}+\tau
\end{aligned}
$$

For more details refer to [10].

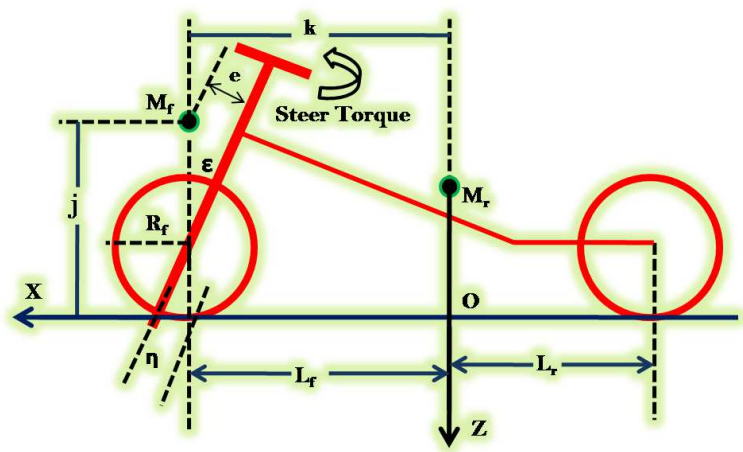

Fig. 3. Schematic Diagram of the Motorcycle

2) Tire Road Interaction: The tire is one of the main components of the vehicle. Indeed, it represents the interface of the aforementioned with the external environment which is the road. It transmits the guidance and braking/tractive efforts [9], [10], [11], [12]. The tire dynamic behavior is very complex and it is linear only under certain restricted conditions of drive. We can observe various phenomena like skidding and blocking.

Ongoing knowledge of this effort duiring the conducts is essential to design preventive security system.

Here, we use the Pacejka model [12] to represent the efforts exerted on each tire. This model is based on the mathematical representation of the tire dynamic behavior using analytical functions having a particular structure. Lateral forces of front and rear tires are function of the sideslip angle $\alpha_{i}$ at the tire-road contact location and the camber angle $\gamma_{i}$. Here, the index $i$ stand for $f$ (front) or $r$ (rear). In this paper, the magic formula of Pacejka, adapted for motorcycle tires, is used for each tire in order to determine the lateral forces [12]:

$$
\begin{aligned}
& F_{i}\left(\alpha_{i}, \gamma_{i}\right)= \\
& d_{i}\left(\gamma_{i}\right) \sin \left(c_{i} \cdot \tan ^{-1}\left(b_{i}\left(\gamma_{i}\right)\left(1-e_{i}\right) \alpha_{i}+e_{i} \cdot \tan ^{-1}\left(b_{i}\left(\gamma_{i}\right) \alpha_{i}\right)\right)\right)
\end{aligned}
$$

The coefficients $b_{i}, c_{i}, d_{i}, e_{i}$ depend on the tire characteristics, on the road conditions and on the vehicle operational conditions.

3) Relaxation model: When vehicle sideslip changes, a lateral tire force is created with a time lag. This transient behavior of tires can be formulated using a relaxation length $\sigma_{i}(i=f, r)$. The first order approximation for front and rear tire is given by:

$$
\left\{\begin{array}{l}
\dot{Y}_{f}=\left(\frac{v_{x}}{\sigma_{f}}\right)\left(-Y_{f}+F_{y f}\right) \\
\dot{Y}_{r}=\left(\frac{v_{x}}{\sigma_{r}}\right)\left(-Y_{r}+F_{y r}\right)
\end{array}\right.
$$

The $F_{y i}$ is a function of the related slip angle, dynamic normal loads and camber angles. These forces are calculated by the Pacejka model.

\section{MODEL TRANSFORMATION}

For the synthesis of the observer, the nonlinear model (2), will be transformed into a linear varying time model (LPV). After transformations, it takes the following form :

$$
E x(t)=A A(t) x(t)+B B u(t)
$$

The state vector $x$ comprises lateral speed at the (cog), roll rate, yaw rate, front and rear lateral forces:

The input vector $u$ comprises the driver steering torque and the lateral tire forces calculated with the magic formula (Pacejka model) given by the first block (see section 2) $x=\left(V_{y}, \dot{\psi}, \dot{\phi}, \dot{\delta}, \phi, \delta\right), u=\left(\tau, F_{y f}, F_{y r}\right)$

The matrix $E E$ is full rank and its determinant is different from zero (i.e $E E^{-1}$ exist). Matrices $A=E E^{-1} A A, B=$ $E E^{-1} B B$ are the state and input matrices (resp), below we give all expressions of these matrices:

$$
E E=\left(\begin{array}{cccccccc}
M & -a_{2} & -a_{1} & -a_{3} & 0 & 0 & 0 & 0 \\
-a_{2} & a_{4} & -a_{5} & -a_{6} & 0 & 0 & 0 & 0 \\
-a_{1} & a_{5} & -a_{10} & -a_{11} & 0 & 0 & 0 & 0 \\
-a_{3} & -a_{6} & -a_{11} & a_{16} & 0 & 0 & 0 & 0 \\
0 & 0 & 0 & 0 & 1 & 0 & 0 & 0 \\
0 & 0 & 0 & 0 & 0 & 1 & 0 & 0 \\
0 & 0 & 0 & 0 & 0 & 0 & 1 & 0 \\
0 & 0 & 0 & 0 & 0 & 0 & 0 & 1
\end{array}\right)
$$

$$
A A(t)=\left(\begin{array}{cccccccc}
d_{11} & d_{12} & 0 & 0 & 0 & 0 & 1 & 1 \\
0 & d_{22} & d_{23} & d_{24} & d_{25} & d_{26} & 0 & 0 \\
0 & d_{22} & 0 & d_{34} & d_{35} & d_{36} & 0 & 0 \\
0 & d_{32} & 0 & d_{34} & d_{35} & d_{36} & 0 & 0 \\
0 & d_{42} & d_{43} & d_{44} & d_{45} & d_{46} & d_{47} & 0 \\
0 & 0 & 1 & 0 & 0 & 0 & 0 & 0 \\
0 & 0 & 0 & 1 & 0 & 0 & 0 & 0 \\
d_{71} & d_{72} & 0 & d_{73} & d_{74} & d_{75} & d_{76} & 0 \\
d_{81} & d_{82} & 0 & 0 & d_{85} & 0 & 0 & d_{88}
\end{array}\right)_{10)}
$$


$d_{11}=-2 C_{y} V_{y}, d_{12}=-M v_{x}, d_{22}=a_{2} v_{x}$

$d_{23}=a_{8} v_{x}, d_{24}=a_{9} v_{x}, d_{82}=\left(\frac{C_{r 1}}{\sigma_{r}}\right) L_{r}$

$d_{26}=-L_{r} \cos (\phi), d_{32}=a_{12} v_{x}, d_{34}=a_{13} v_{x}$

$d_{35}=a_{15} v_{x} \cos (\phi), d_{88}=-\left(\frac{v_{x}}{\sigma_{r}}\right), d_{81}=-\left(\frac{C_{r 1}}{\sigma_{r}}\right)$

$d_{36}=a_{14} \cos (\delta), d_{47}=-, d_{71}=-\left(\frac{C_{f 1}}{\sigma_{f}}\right)$

$d_{42}=a_{17} v_{x}, d_{43}=-a_{13} v_{x}, d_{44}=-K_{\delta}$

$d_{45}=a_{14} \cos (\phi), d_{75}=\left(\frac{v_{x}}{\sigma_{f}}\right) C_{f 2}$

$d_{46}=a_{18} \cos (\delta), d_{25}=-\left(L_{f} Y_{f}-L_{r} Y_{r}\right) \sin (\phi) L_{f} \cos (\phi)$

$d_{72}=-\left(\frac{C_{f 1}}{\sigma_{f}}\right) L_{f}, d_{74}=\left(\frac{C_{f 1}}{\sigma_{f}}\right)$

$d_{76}=\left(\frac{v_{x}}{\sigma_{f}}\right)\left(\sin (\varepsilon) C_{f 2}+C_{f 1} \cos (\varepsilon)\right)$

$d_{77}=-\left(\frac{v_{x}}{\sigma_{f}}\right), d_{82}=\left(\frac{v_{x}}{\sigma_{r}}\right) C_{r 2}$

$$
B B^{T}=\left(\begin{array}{llllllll}
0 & 0 & 0 & 1 & 0 & 0 & 0 & 0
\end{array}\right)
$$

Now, the new system becomes:

$$
\dot{x}(t)=A(t) x(t)+B u(t)
$$

a) Remark $1:$ The motorcycle is modeled using a lateral model with constant longitudinal speed, but in reality this last is considered as variable parameter. To ensure the robustness of the observer with respect to this parameter, we conducted several tests at variable speed (see section of the simulation results).

\section{OBSERVER DESIGN}

We develop in this section an observer based sliding mode technique to estimate the lateral dynamic of the motorcycle. This method estimation is designed using a dynamic model of the vehicle and based on all measures: yaw rate, roll rate, roll and yaw angles.

The sliding mode observer, has the distinction of being robust compared to modeling errors, parameter uncertainties and external disturbances [13]. This section presents a description of the observer dedicated to the observation method.

We recall above the linear stochastic state-space representation of the system described before by:

$$
\left\{\begin{array}{l}
\dot{x}(t)=A(t) x(t)+B u(t)+b_{m}(t) \\
y(t)=C x(t)+b_{s}(t)
\end{array}\right.
$$

Observation matrix is chosen as follows:

$$
C=\left(\begin{array}{llllllll}
0 & 1 & 0 & 0 & 0 & 0 & 0 & 0 \\
0 & 0 & 1 & 0 & 0 & 0 & 0 & 0 \\
0 & 0 & 0 & 1 & 0 & 0 & 0 & 0 \\
0 & 0 & 0 & 0 & 0 & 1 & 0 & 0
\end{array}\right)
$$

The measure vector $y(t)$ comprises yaw rate, roll rate and steering rate:

$$
y=(\dot{\psi}, \dot{\phi}, \phi, \delta)=\left(y_{1}, y_{2}, y_{3}, y_{4}\right)
$$

$x=\left(V_{y}, \dot{\psi}, \dot{\phi}, \dot{\delta}, \phi, \delta, Y_{f}, Y_{r}\right)=\left(x_{1}, x_{2}, x_{3}, x_{4}, x_{5}, x_{6}, x_{5}, x_{6}, x_{7}, x_{8}\right)$

The process and measurement noise vectors, respectively $b_{m}(t)$ and $b_{s}(t)$, are assumed to be white, zero mean and uncorrelated.

The state vector $x(t)$ will be estimated by applying the slidind mode observer, for which we give the general form as shown in figure.4: $H$ is the observer gain. It is calculated

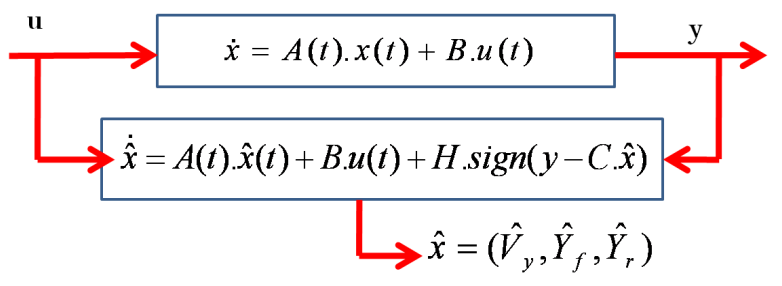

Fig. 4. Diagram of Sliding Mode Observer

so that the eigenvalues of the matrix $(A(t)-H C)$ are negative real parts, that is to say that the observer is stable and that the dynamics of the observer is sufficiently fast to that of the system.

The purpose of an observer is to converge the estimated state to the true value of the state in established regim (i.e $\lim (x-$ $\hat{x})=0$ ).

It has come back to find a positive definite gain matrix, here called $H$.

$H$ in our case is synthesized using the pole placement technique.

4) Observability: Observability is a measure of how well the internal states of a system can be inferred from knowledge of its inputs and external outputs. This property is often presented as a rank condition on the observability matrix. Using the linear state space withe varying time formulation (LPV) of the system represented in 6), the observability definition is local.

Analysis of observability of the system has been shown that the system is observable except when:

- steering torque are null,

- vehicle is at rest $\left(v_{x}=0\right)$.

The determinant of the observability matrix $O=[C, C A]$ is nonzero except in previous particular cases.

For these situations, we assume that lateral forces and lateral speed are null, which approximately corresponds to the real cases.

5) Estimation method: The aim of an observer or a virtual sensor is to estimate a particular unmeasurable variable from available measurements and a system model in a closed loop observation scheme. A simple example of an open loop observer is the model given by relations (2) through (4). Because of the system-model mismatch (unmodelled dynamics, parameter variations, ... ) and the presence of unknown and unmeasurable disturbances, the calculation obtained from the open loop observer would deviate from the actual values over time. In order to reduce the estimation error, at least some of the measured outputs are compared to the same variables estimated by the observer. The difference is feed back into the observer after being multiplied by a gain matrix 
$H$, and so we have a closed loop observer. The observer was implemented in a first-order Euler approximation. At each iteration, the state vector is first calculated according to the evolution equation and then corrected online with the measurement errors. The gain is calculated using the sliding mode method which is a set of mathematical equations and is widely used in literature due to its rubustesse.

6) Simulation Results: In this section, we present some results of estimating process.

We also analyze on the considered scenario, the performance of estimations compared to speed variation and initial conditions.

The results of motorcycle states estimation and the reconstruction of the lateral dynamics are presented. The steering torque $(\tau)$ applied to the forward direction is shown in figure.5, it represents a double lane change (chicane). In this section simulation, the road is in good adhesion (the coefficients of adhesion $(\mu)$ are assumed to be constant and fixed at about 0.7). Recall that, the speed of vehicle is constant and is set around $25 \mathrm{~m} / \mathrm{s}$.

The vehicle is initially considered in a straight line. At time $t=5 \mathrm{~s}$, the vehicle covers a portion of negative clothoid, it moves later on a portion of an arc at $t=14 \mathrm{~s}$, before resuming a clothoid opposite curvature at $t=16 \mathrm{~s}$ which brings on a straight line at $t=25 \mathrm{~s}$. At $t=35 \mathrm{~s}$ the vehicle takes the opposite path, finishing at $t=60 \mathrm{~s}$. The road is assumed flat (no elevation or slope). The scenario chosen is a double lane change on a flat road.

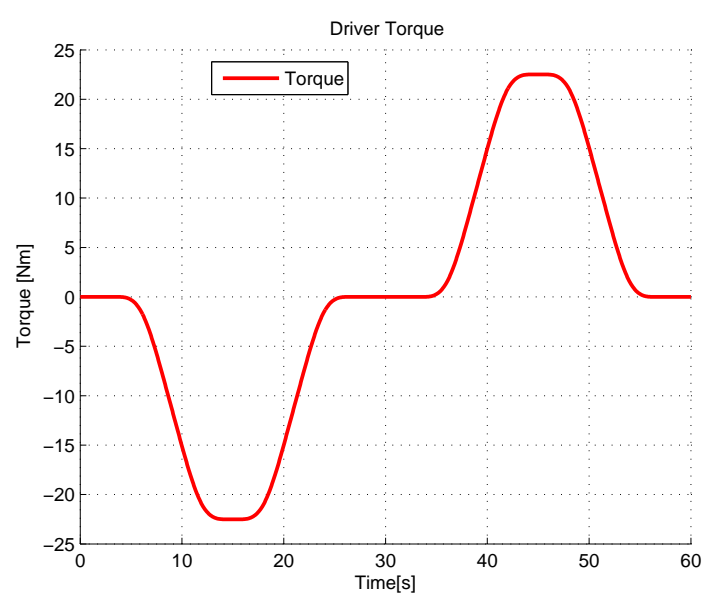

Fig. 5. Driver Steering Torque

\section{A. Observer parameters}

The sample time of variables (inputs and measured outputs) is fixed at $0.083(120 \mathrm{~Hz})$. The errors of the sensors are set according to the variation range of measured data:

$$
\sqrt{\sigma_{\dot{\psi}}}=0.01, \sqrt{\sigma_{\dot{\phi}}}=0.01, \sqrt{\sigma_{\phi}}=0.01, \sqrt{\sigma_{\delta}}=0.001 .
$$

The observability of the system is checked for any non-zero longitudinal speed. figure. 6 and figure7. shows the estimation results for the lateral velocity, the steering rate of the direction, the front side force and rear side force. These obtained curves, as well as estimation errors presented in figure. 8 and figure.9, allow concluding that these variables are perfectly estimated.

\section{B. Robustness of the observer against initial conditions}

Assuming that the initial conditions of the observer variables are not zero, repeating the same scenario as before, the figures.10 and 11, show the simulation results of different state in this case. It may be noted that the estimate state is correct and joined the model after a short transition period due to the initial conditions.
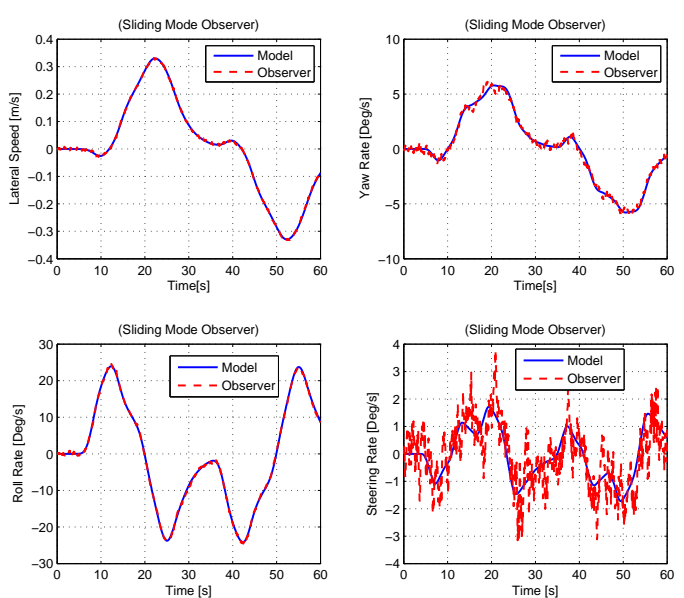

Fig. 6. Lateral Speed, Yaw Rate, Roll Rate and Steering Rate Estimation
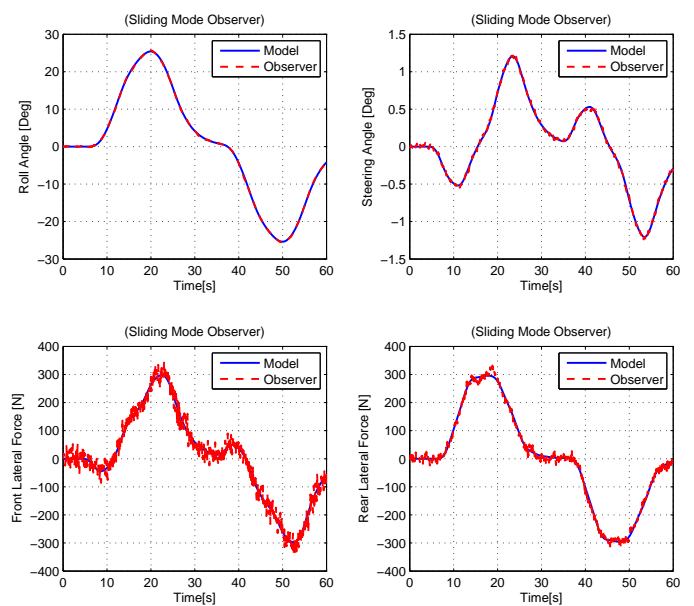

Fig. 7. Roll Angle, Steering Angle, Front Side and Rear Side Forces Estimation

C. Robustness of the observer against forward speed variation

As stated in previous sections, the forward velocity in the model is considered as fixed parameter, it is not quite true, 

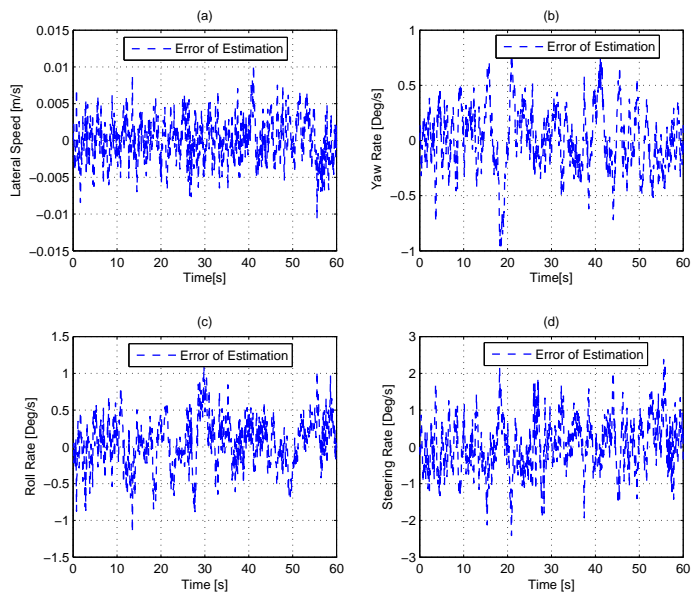

Fig. 8. Error Estimation on Lateral Speed, Yaw Rate, Roll Rate and Steering Rate
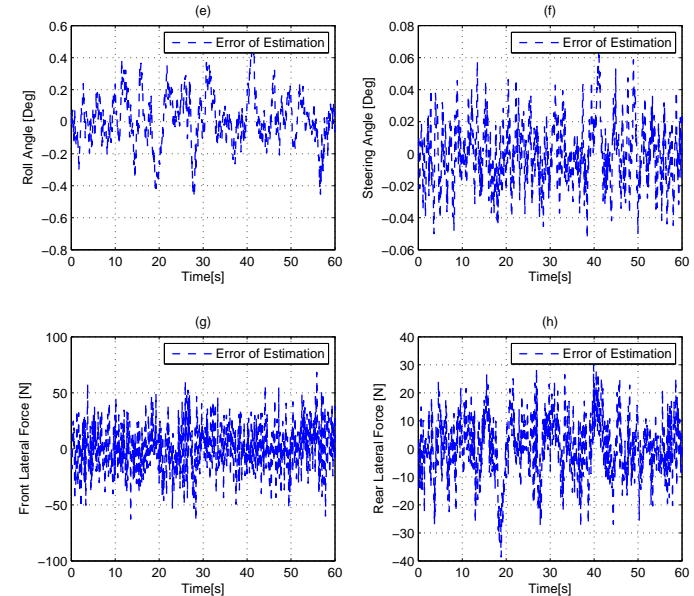

Fig. 9. Error Estimation on Roll Angle, Steering Angle, Front and Rear Side Forces

since this last is a varying parameter.

For the same simulation conditions, the figures.12 and 13, show the new estimate of states at different forward speed $(18,20,22,24,25 \mathrm{~m} / \mathrm{s})$, and errors caused by reconstruction remain relatively low, again we can conclude on a good estimate and therefore the robustness of the observer against this parameter.

\section{CONClusions ANd Future Works}

This paper has presented a new method for estimating lateral tire forces and lateral speed, that is to say two of the most important parameters affecting motorcycle stability and the risk of loss control. The developed observer is derived from a four D-O-F motorcycle model and is based on sliding mode observer technique. Tire-road interaction is represented by the Pacejka model. Moreover, the robustness of the observer with respect to variations of the forward speed and different initial conditions has been shown.
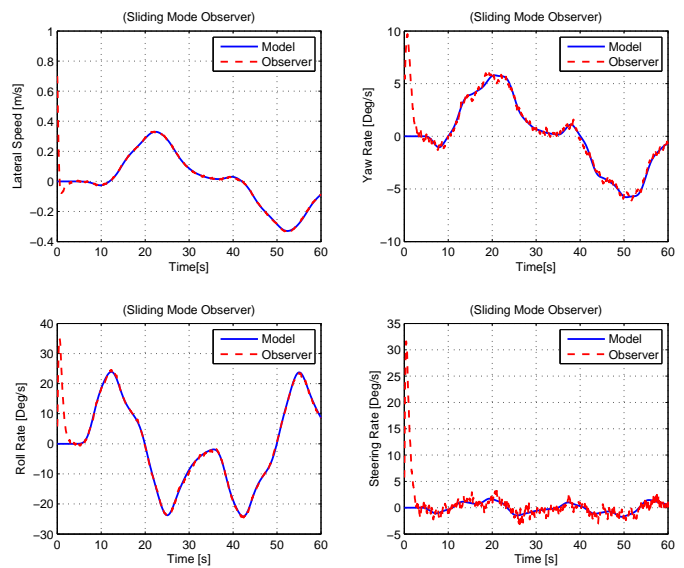

Fig. 10. Lateral Speed, Yaw Rate, Roll Rate and Steering Rate with nonzero Initial Conditions
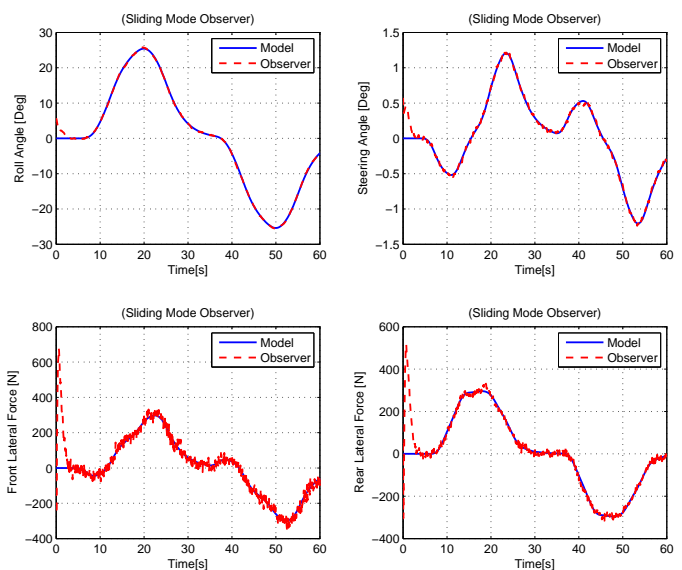

Fig. 11. Roll Angle, Steering Angle, Front and Rear Side Forces Estimation with nonzero Initial Conditions

Simulations test demonstrates the potential of the estimation process, showing that it may be possible to replace expensive sensors by software observers that can work in real-time while the vehicle is in motion.

This is one of the important results of our work. Another important result concerns the estimation of individual lateral forces acting on each tire of the motorcycle, that is an evolution with respect to the current literature concerning the vehicle dynamic community. Future studies will improve vehicle/road model in order to widen validity domains for the observer. Subsequent, vehicle/road models will take into account longitudinal and vertical dynamics. Moreover, it will be of paramount importance to study the effect of the coupling longitudinal/lateral dynamics on lateral side forces behavior.

\section{REFERENCES}

[1] F. Aparicio et al, "Discussion of a new adaptive speed control system incorporating the geometric characteristics of the road" Int. J. Vehicle Autonomous Systems, vol.3, No.1, pp.47-64, 2005. 

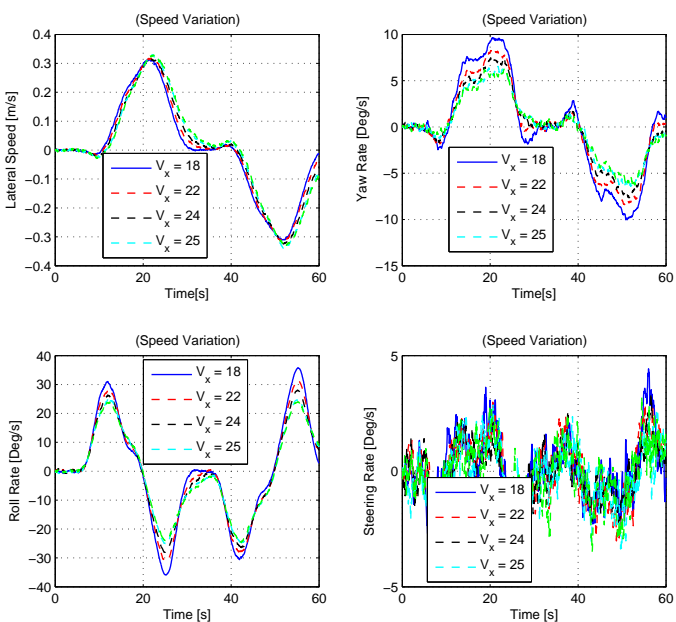

Fig. 12. Laterel Speed, Yaw Rate, Roll Rate and Steering Rete Estimation at variable speeds
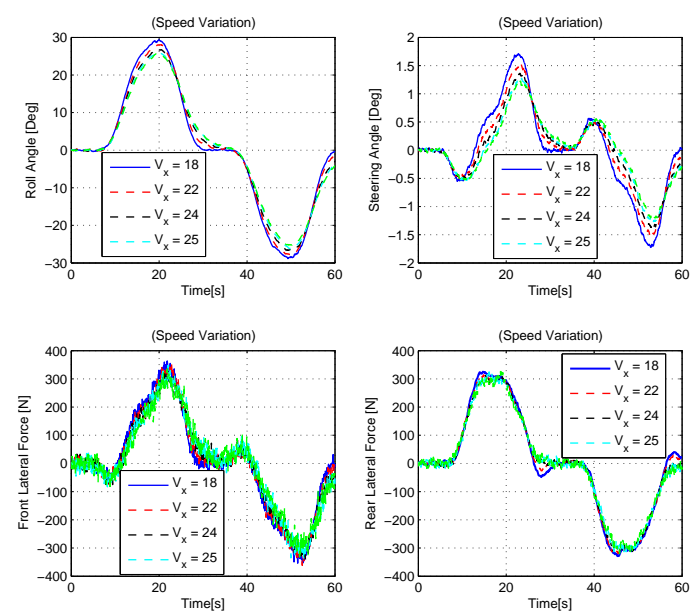

Fig. 13. Roll Angle, Steering Angle, Front Side and Rear Side Forces Estimation at variable speeds

[2] L. R. Ray, "Nonlinear tire force estimation and road friction identification : simulation and experiments" Automatica, vol. 33, no. 10, pp. 1819-1833, 1997.

[3] H. Slimi, H. Arioui, L. Nouveliere and S. Mammar, "Advanced Motorcycle-Infrastructure-Driver Roll Angle Profile for Loss Control Prevention", 12th International IEEE Conference on Intelligent Transportation Systems., St. Louis, Missouri, U.S.A., October 3-7, 2009

[4] J. Stphant, A. Charara and D. Meizel, "Virtual sensor, application to vehicle sideslip angle and transversal forces" IEEE Transactions on Industrial Electronics, vol. 51, no. 2, April 2004.

[5] U. Kiencke and L. Nielsen, "Automotive control systems", Springer, 2000.

[6] G. Baffet A. Charara, D. Lechner and D. Thomas, "Experimental evaluation of observers for tire-road forces, sideslip angle and wheel cornering stiffness" VSD, vol. 45, pp.191-216, june 2008.

[7] B. Amans, M. Moutreuil, "RIDER : Recherche sur les accidents impliquant un deux roues motorisé", Reaserch Report of RIDER Project, Mars 2005.

[8] D. J. N. Limebeer and R. S. Sharp, "Bicycles, motorcycles and models", IEEE Control Systems magazine, vol. 26, no. 5, pp. 34-61, 2006.

[9] R. S. Sharp and D. J. N. Limebeer, "A motorcycle model for stability

and control analysis", Multibody System Dynamics, vol. 6, no. 2, pp. 123-142, 2001.

[10] V. Cossalter and R. Lot, "A motorcycle multi-body model for real time simulations based on the natural coordinates approach", Vehicle System Dynamics, vol. 37, no. 6, pp. 423-447, 2002.

[11] H. Slimi, H. Arioui, L. Nouveliere and S. Mammar, "Preventive Safety: Warning System for Control Loss of Two-Wheeled Vehicles", Informatics, Integrative Biology and Complex Systems, Zarzis, Tunisia on 23-25 March 2009.

[12] Hans B. Pacejka, "Tyre and Vehicle Dynamics", p 511- 562 , Delft University of Technology, 2002.

[13] J. P. Barbot, T. Boukhobza, M. Djemai, "Triangular Input Observer Form and Sliding Mode Observer", In In IEEE Conf. On Decision and Control, pages 14891491, 1996.

$\phi, \psi, \delta$

$M, M_{f}, M_{r}$

$\phi_{p}, \phi_{r}$

$\tau, \eta, \varepsilon$

$j, k, h, L_{f}, L_{r}, e$

\section{APPENDIX}

Roll, yaw and direction angles Total, front and rear masses Slope and road bank angles Steering torque, Trail, Caster angle dimensional parameters (Figure 2) 